\title{
Microketides A and B, Polyketides from a Gorgonian-Derived Microsphaeropsis sp. Fungus
}

Yun-Feng Liu, ${ }^{\dagger, \star}$ Ya-Hui Zhang, ${ }^{\dagger, \$}$ Chang-Lun Shao, ${ }^{\dagger, \S}$ Fei Cao,,$* *$ Chang-Yun Wang ${ }^{\dagger, \S, *}$

†Key Laboratory of Marine Drugs, the Ministry of Education of China, School of Medicine and Pharmacy; Institute of Evolution \& Marine Biodiversity, Ocean University of China, Qingdao 266003, People's Republic of China

\#College of Pharmaceutical Sciences, Hebei University, Baoding 071002, People's Republic of China

${ }^{\S}$ Laboratory for Marine Drugs and Bioproducts, Qingdao National Laboratory for Marine Science and Technology, Qingdao 266237, People's Republic of China

*Correspondence and requests for materials should be addressed to F. C. (caofei542927001@163.com) or C.-Y.W. (changyun@ouc.edu.cn) 


\section{List of Supporting Information}

Figure S1. ${ }^{1} \mathrm{H}$ NMR $\left(500 \mathrm{MHz}, \mathrm{CD}_{3} \mathrm{OD}\right)$ spectrum of compound 1

Figure S2. ${ }^{13} \mathrm{C}$ NMR (125 MHz, $\left.\mathrm{CD}_{3} \mathrm{OD}\right)$ spectrum of compound 1

Figure S3. $\mathrm{HSQC}\left(\mathrm{CD}_{3} \mathrm{OD}\right)$ spectrum of compound 1

Figure S4. $\mathrm{COSY}\left(\mathrm{CD}_{3} \mathrm{OD}\right)$ spectrum of compound 1

Figure S5. $\mathrm{HMBC}\left(\mathrm{CD}_{3} \mathrm{OD}\right)$ spectrum of compound 1

Figure S6. 1D NOE (500 MHz, $\left.\mathrm{CD}_{3} \mathrm{OD}\right)$ spectrum of compound 1

Figure S7. 1D NOE (500 MHz, $\mathrm{CD}_{3} \mathrm{OD}$ ) spectrum of compound 1

Figure S8. HRESIMS spectrum of compound 1

Figure S9. ${ }^{1} \mathrm{H}$ NMR $\left(500 \mathrm{MHz}, \mathrm{CD}_{3} \mathrm{OD}\right)$ spectrum of compound $\mathbf{1 r}$

Figure S10. ${ }^{1} \mathrm{H}$ NMR $\left(500 \mathrm{MHz}, \mathrm{CD}_{3} \mathrm{OD}\right)$ spectrum of compound $1 \mathrm{~s}$

Figure S11. ESIMS spectrum of compound 1r

Figure S12. ESIMS spectrum of compound 1s

Figure S13. ${ }^{1} \mathrm{H}$ NMR ( $\left.500 \mathrm{MHz}, \mathrm{CD}_{3} \mathrm{OD}\right)$ spectrum of compound 2

Figure S14. ${ }^{13} \mathrm{C}$ NMR $\left(125 \mathrm{MHz}, \mathrm{CD}_{3} \mathrm{OD}\right)$ spectrum of compound 2

Figure S15. HSQC $\left(\mathrm{CD}_{3} \mathrm{OD}\right)$ spectrum of compound 2

Figure S16. $\mathrm{COSY}\left(\mathrm{CD}_{3} \mathrm{OD}\right)$ spectrum of compound 2

Figure S17. $\mathrm{HMBC}\left(\mathrm{CD}_{3} \mathrm{OD}\right)$ spectrum of compound $\mathbf{2}$

Figure S18. 1D NOE (500 MHz, $\left.\mathrm{CD}_{3} \mathrm{OD}\right)$ spectrum of compound 2

Figure S19. 1D NOE (500 MHz, $\mathrm{CD}_{3} \mathrm{OD}$ ) spectrum of compound 2

Figure S20. HRESIMS spectrum of compound 2

Table S1. Experimental and calculated ${ }^{13} \mathrm{C}$ NMR data of $\mathbf{1}$ and $\mathbf{2}$

Table S2 The data of DP4+ method of compounds 1 and $\mathbf{2}$

Table S3. The coordinate for the lowest-energy conformer $[(10 S, 11 S)-1]$ in ECD and ${ }^{13} \mathrm{C}$ NMR calculations

Table S4. The coordinate for the lowest-energy conformer $[(10 S, 11 R)-2]$ in ECD and ${ }^{13} \mathrm{C}$ NMR calculations

Table S5. The coordinate for the lowest-energy conformer $[(10 R, 11 S)-\mathbf{1}]$ in ECD calculation

Table S6. The coordinate for the lowest-energy conformer $[(10 R, 11 R)-1]$ in ECD calculation 


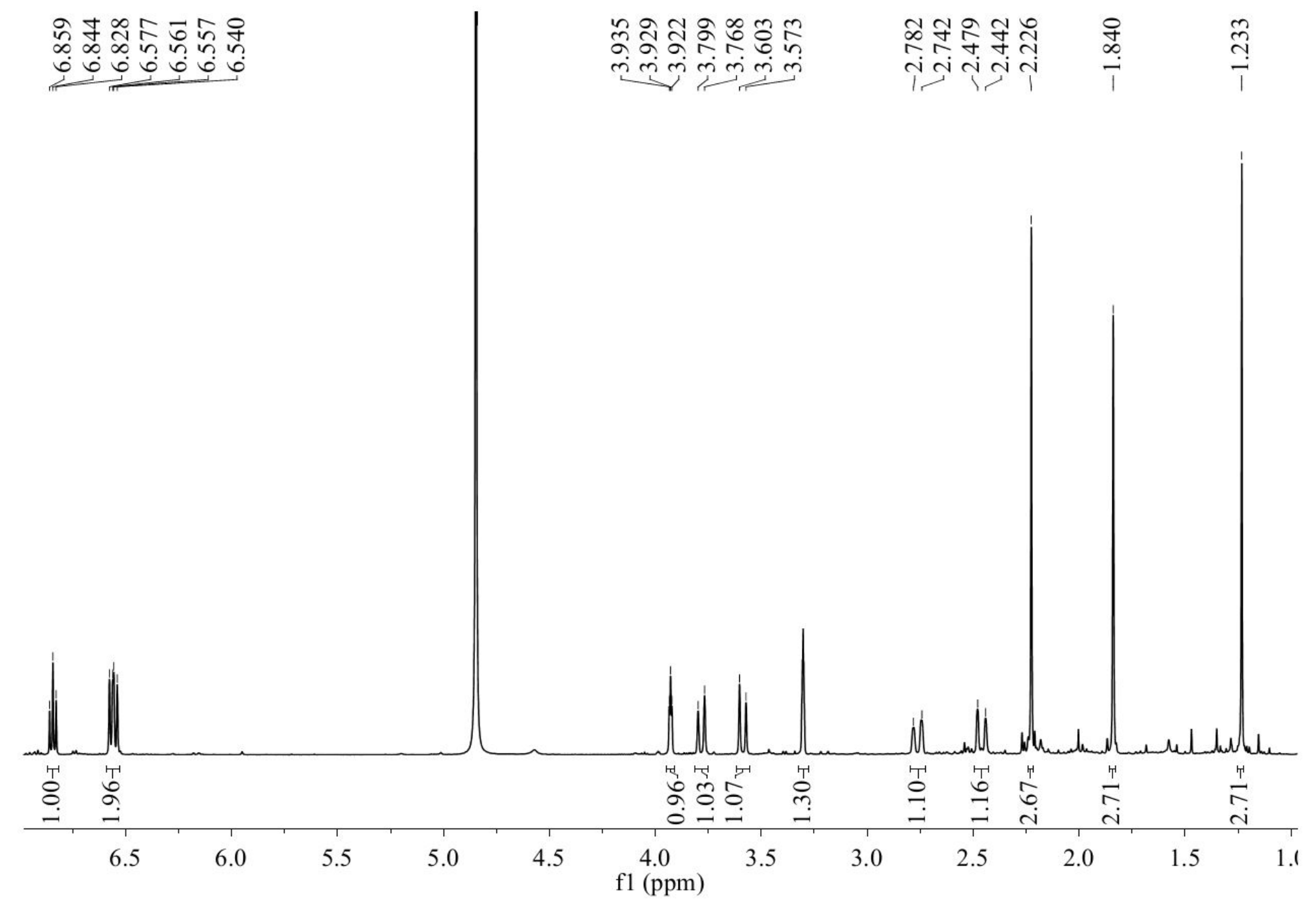

Figure S1. ${ }^{1} \mathrm{H}$ NMR $\left(500 \mathrm{MHz}, \mathrm{CD}_{3} \mathrm{OD}\right)$ spectrum of compound 1 


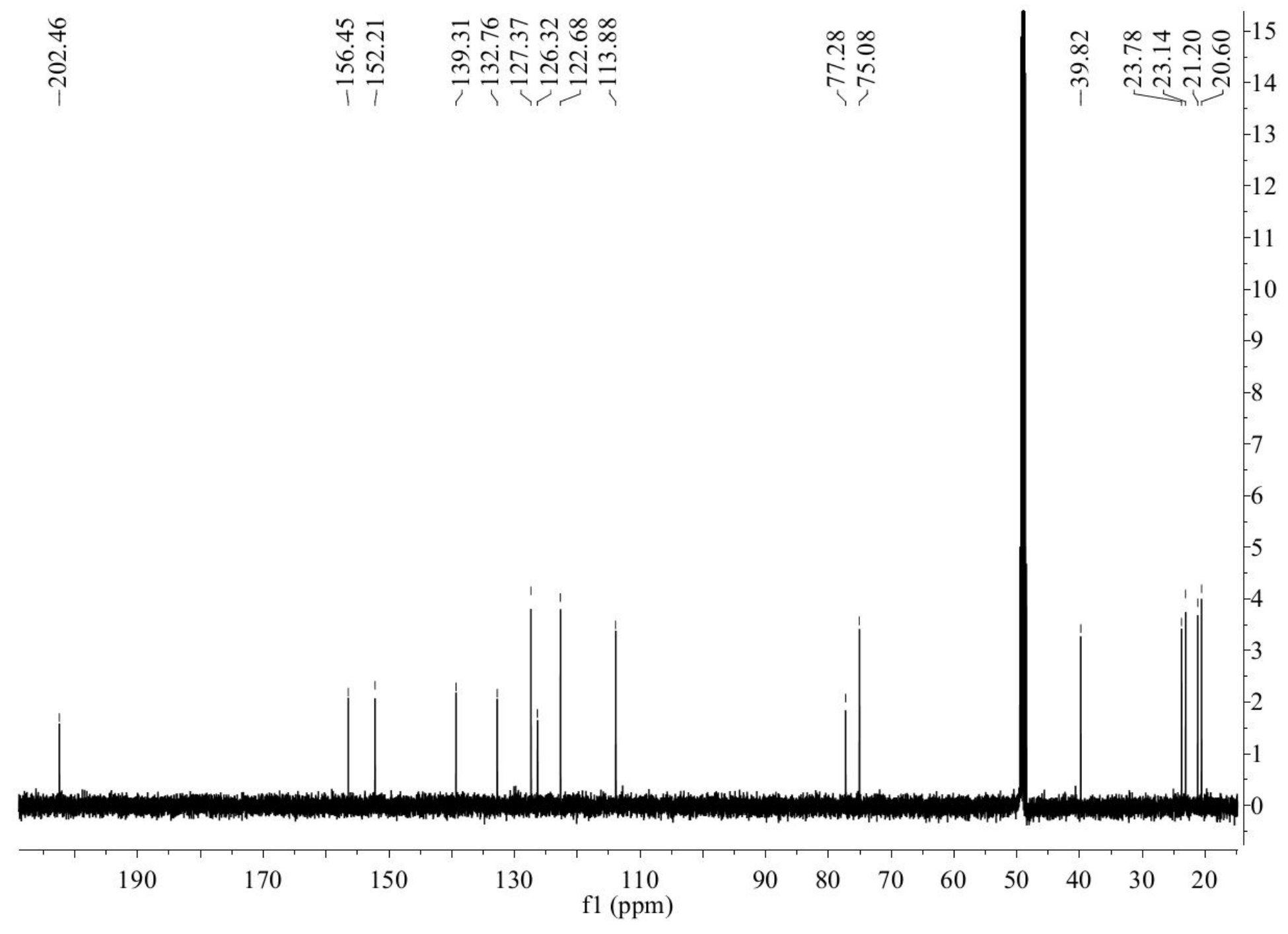

Figure S2. ${ }^{13} \mathrm{C}$ NMR (125 MHz, $\mathrm{CD}_{3} \mathrm{OD}$ ) spectrum of compound $\mathbf{1}$ 


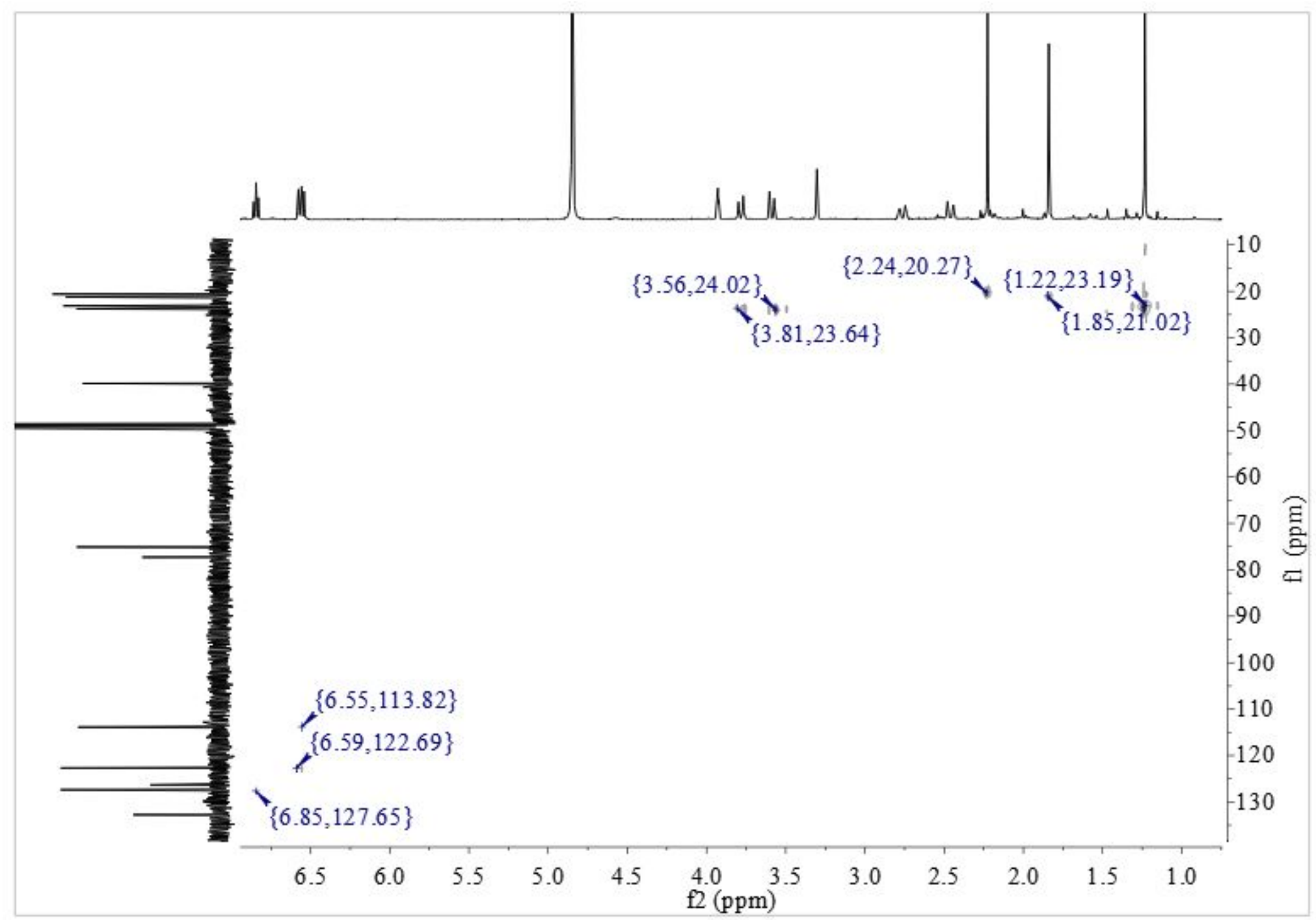

Figure S3. HSQC $\left(\mathrm{CD}_{3} \mathrm{OD}\right)$ spectrum of compound $\mathbf{1}$ 


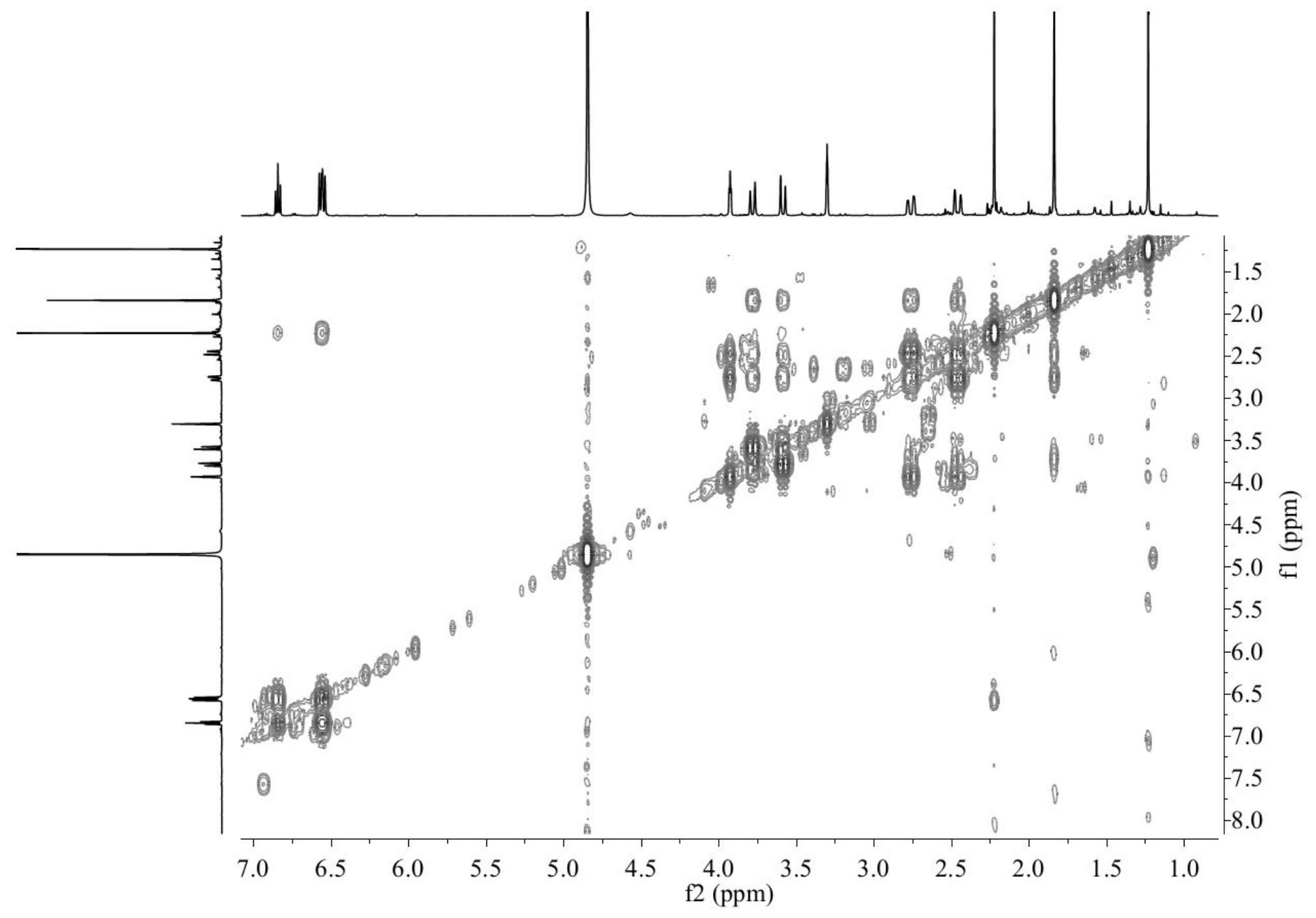

Figure S4. $\mathrm{COSY}\left(\mathrm{CD}_{3} \mathrm{OD}\right)$ spectrum of compound $\mathbf{1}$ 


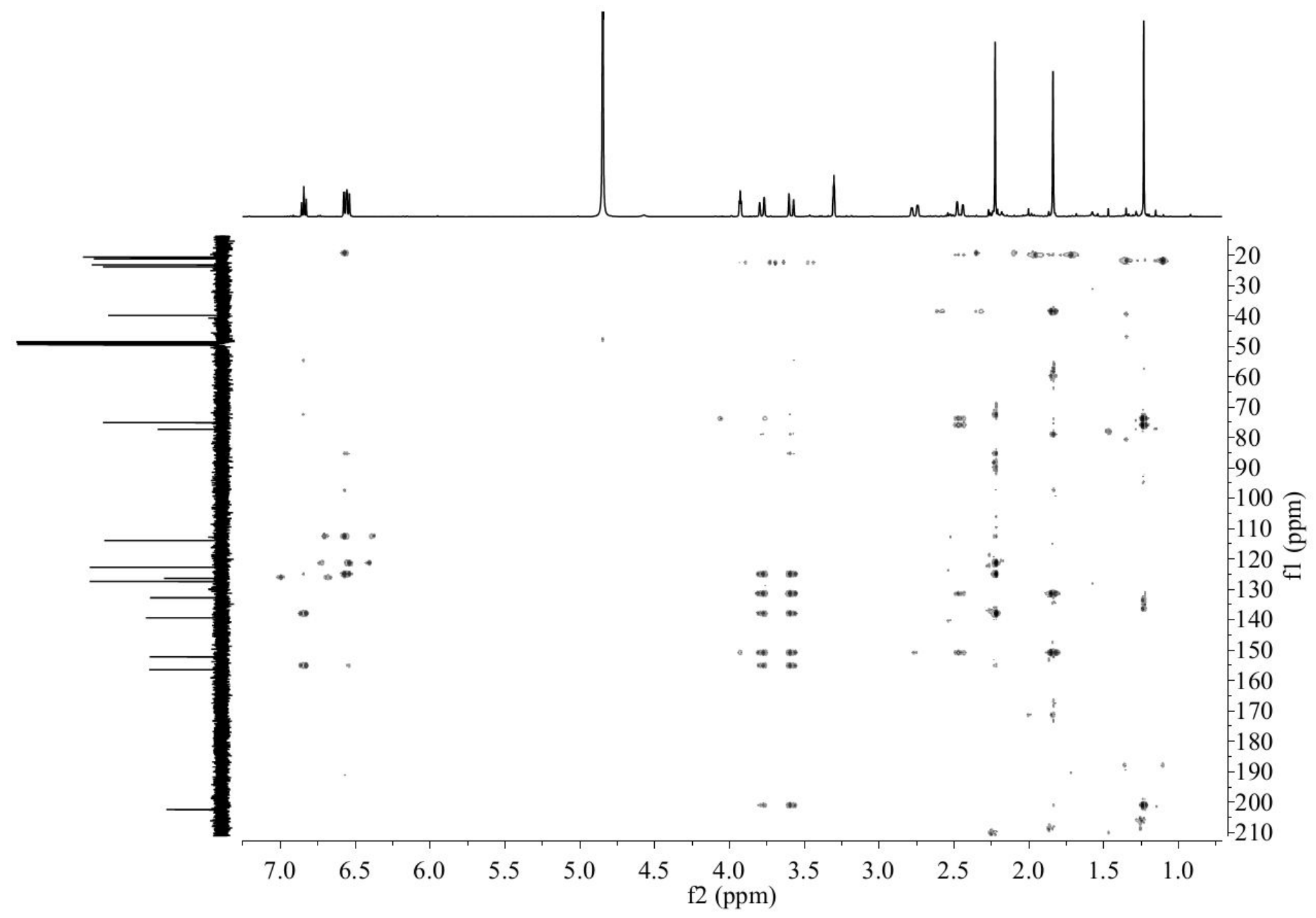

Figure S5. HMBC $\left(\mathrm{CD}_{3} \mathrm{OD}\right)$ spectrum of compound 1 


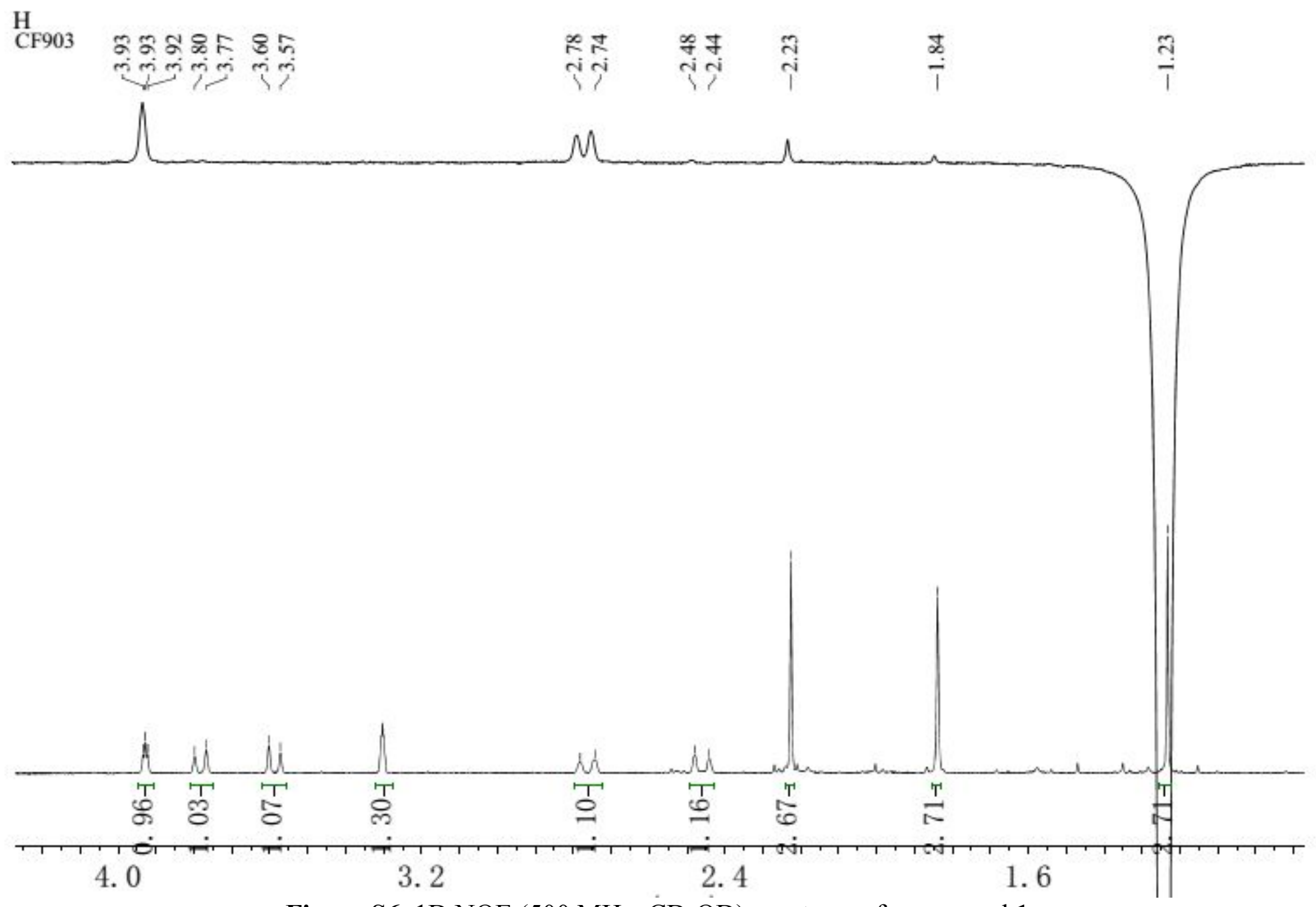

Figure S6. 1D NOE (500 MHz, $\left.\mathrm{CD}_{3} \mathrm{OD}\right)$ spectrum of compound 1 


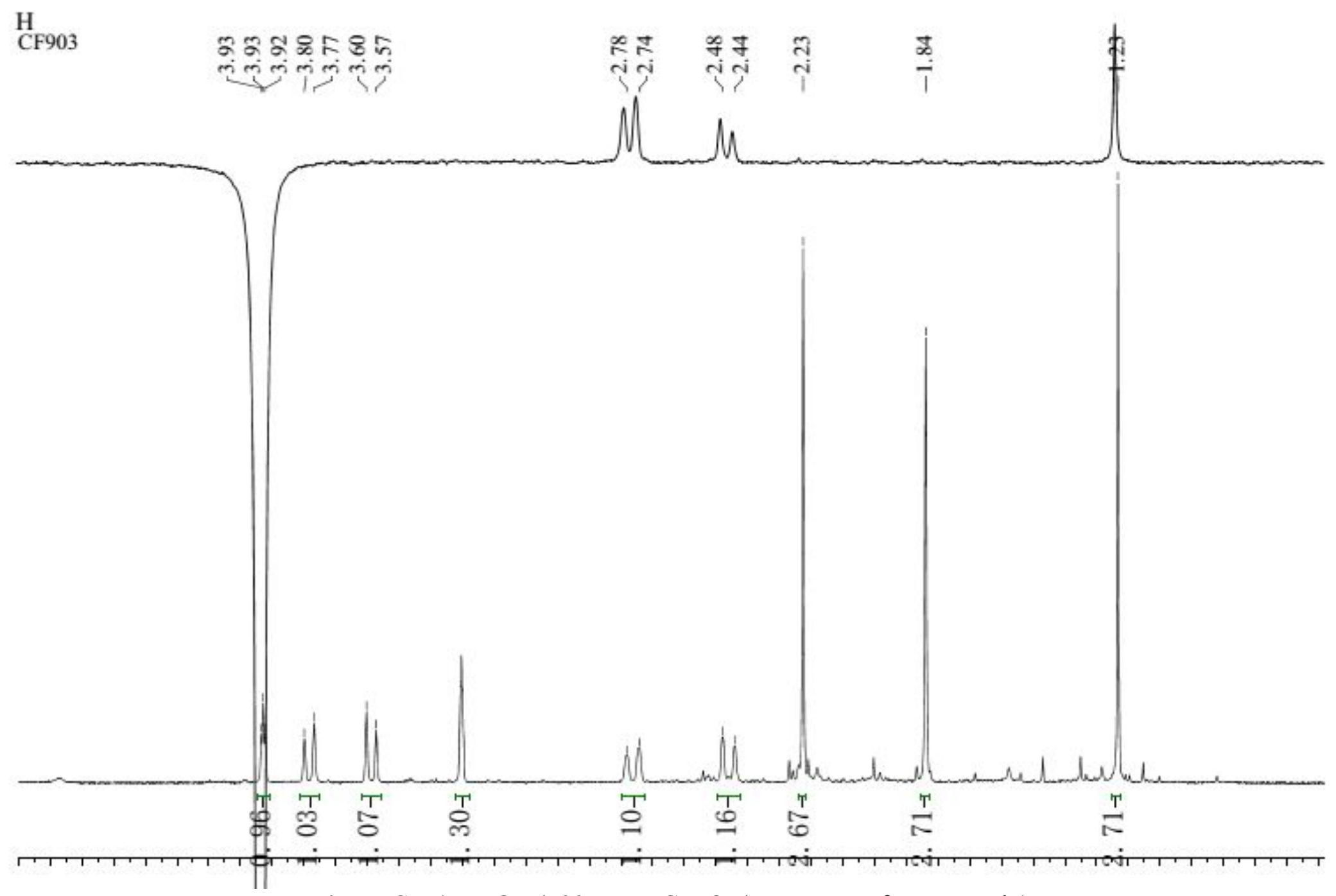

Figure S7. 1D NOE (500 MHz, $\left.\mathrm{CD}_{3} \mathrm{OD}\right)$ spectrum of compound 1 


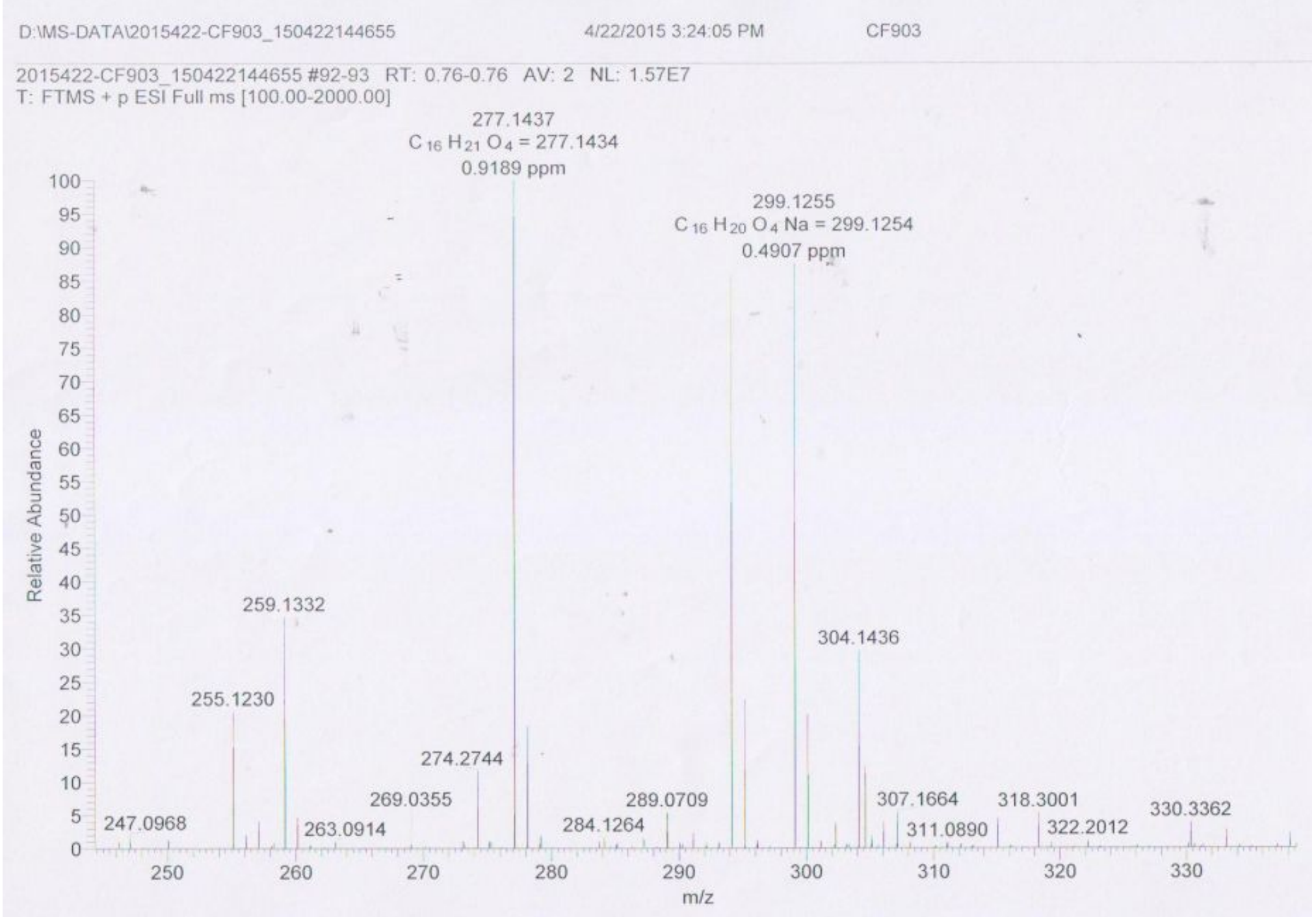

Figure S8. HRESIMS spectrum of compound 1 


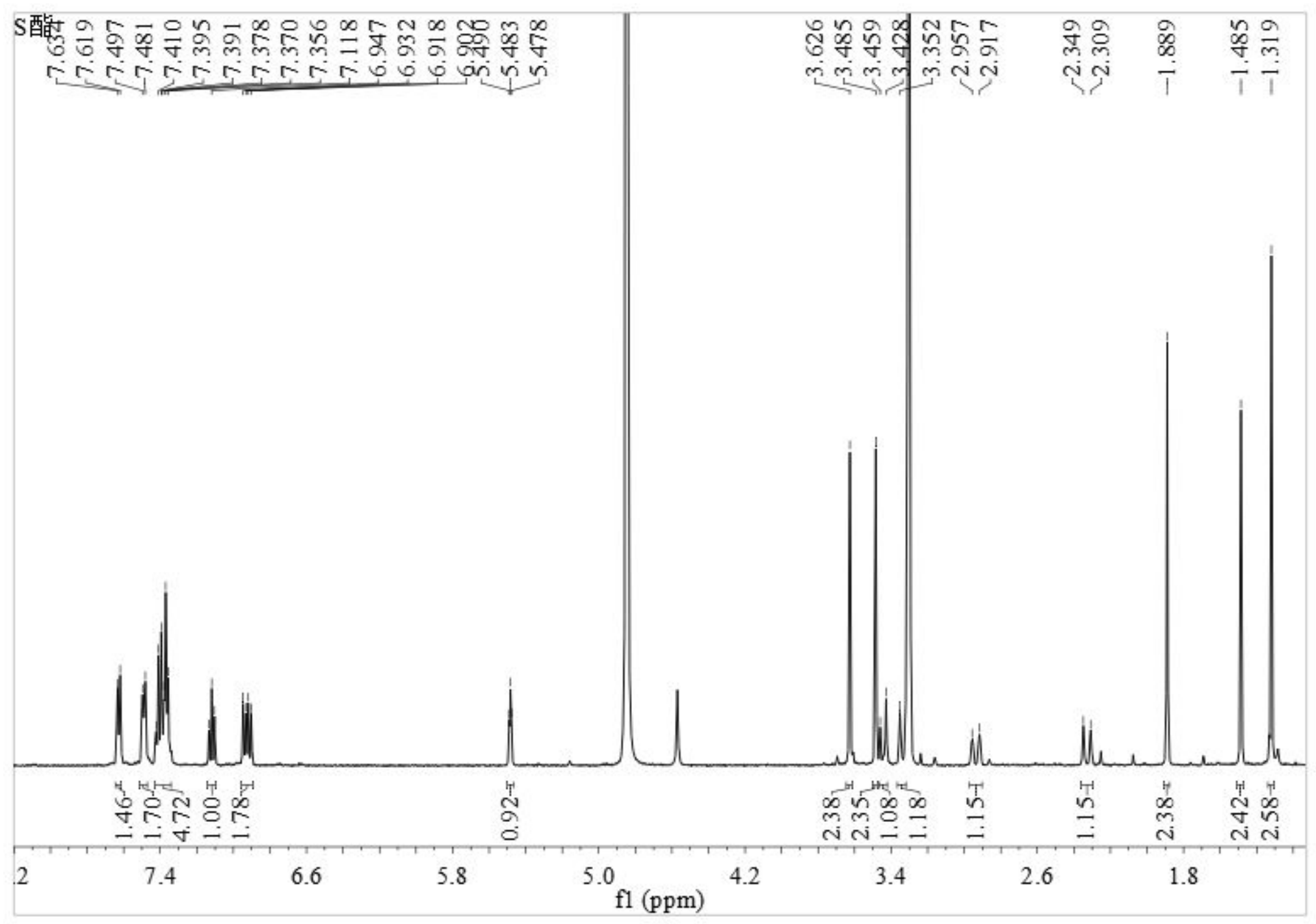

Figure S9. ${ }^{1} \mathrm{H} \mathrm{NMR}\left(500 \mathrm{MHz}, \mathrm{CD}_{3} \mathrm{OD}\right)$ spectrum of compound $1 \mathbf{r}$ 


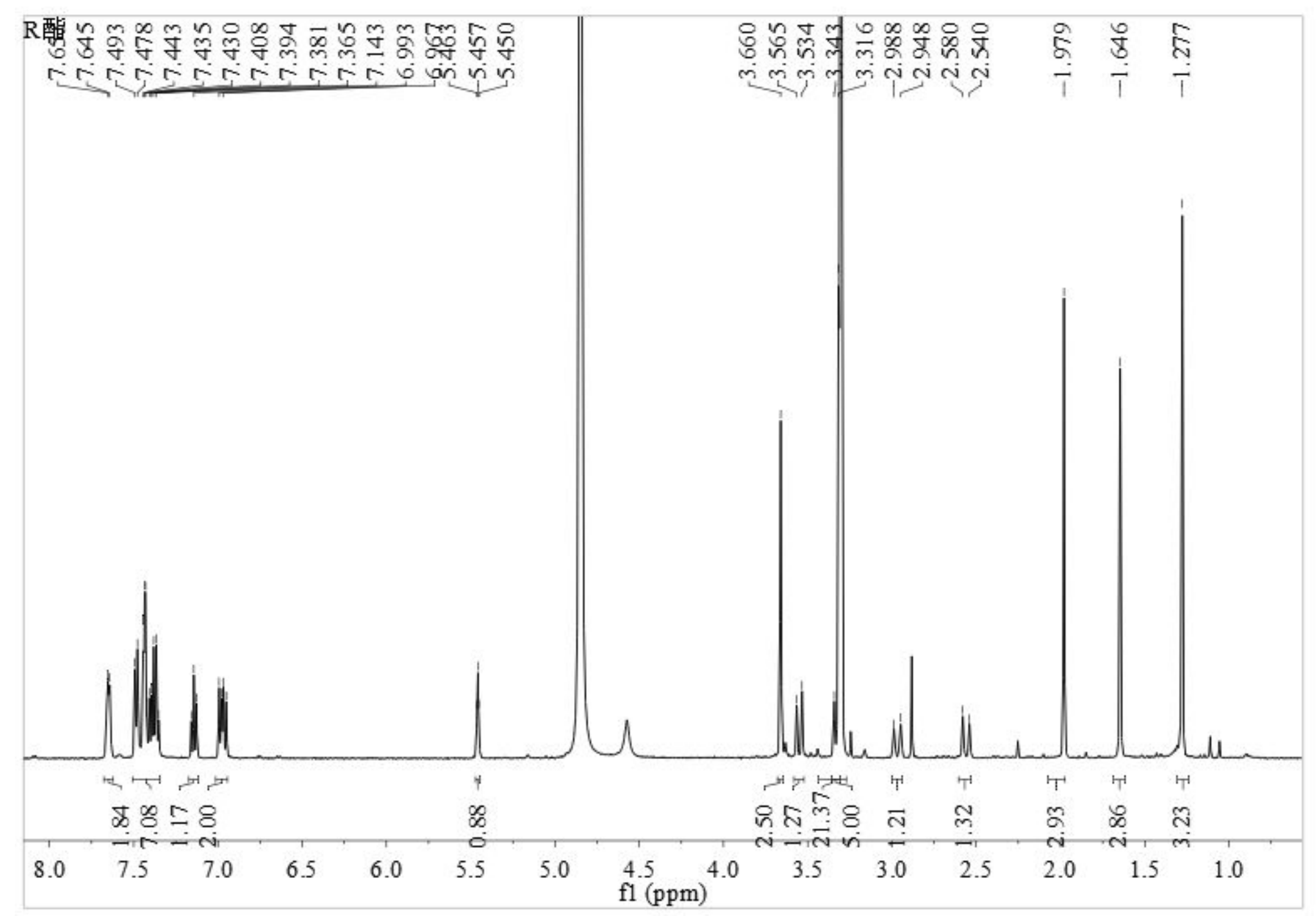

Figure S10. ${ }^{1} \mathrm{H}$ NMR $\left(500 \mathrm{MHz}, \mathrm{CD}_{3} \mathrm{OD}\right)$ spectrum of compound $1 \mathrm{~s}$ 


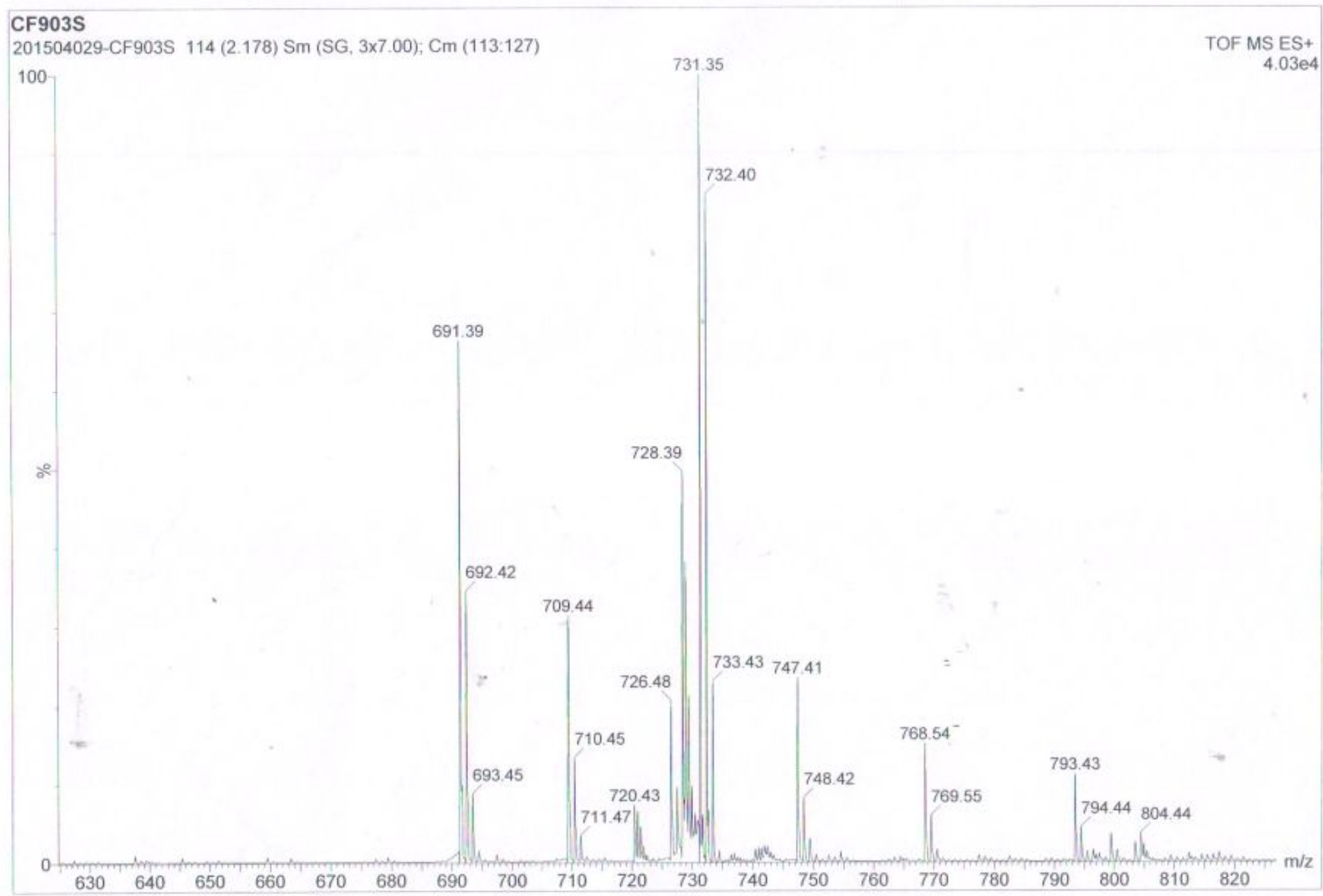

Figure S11. ESIMS spectrum of compound 1r 


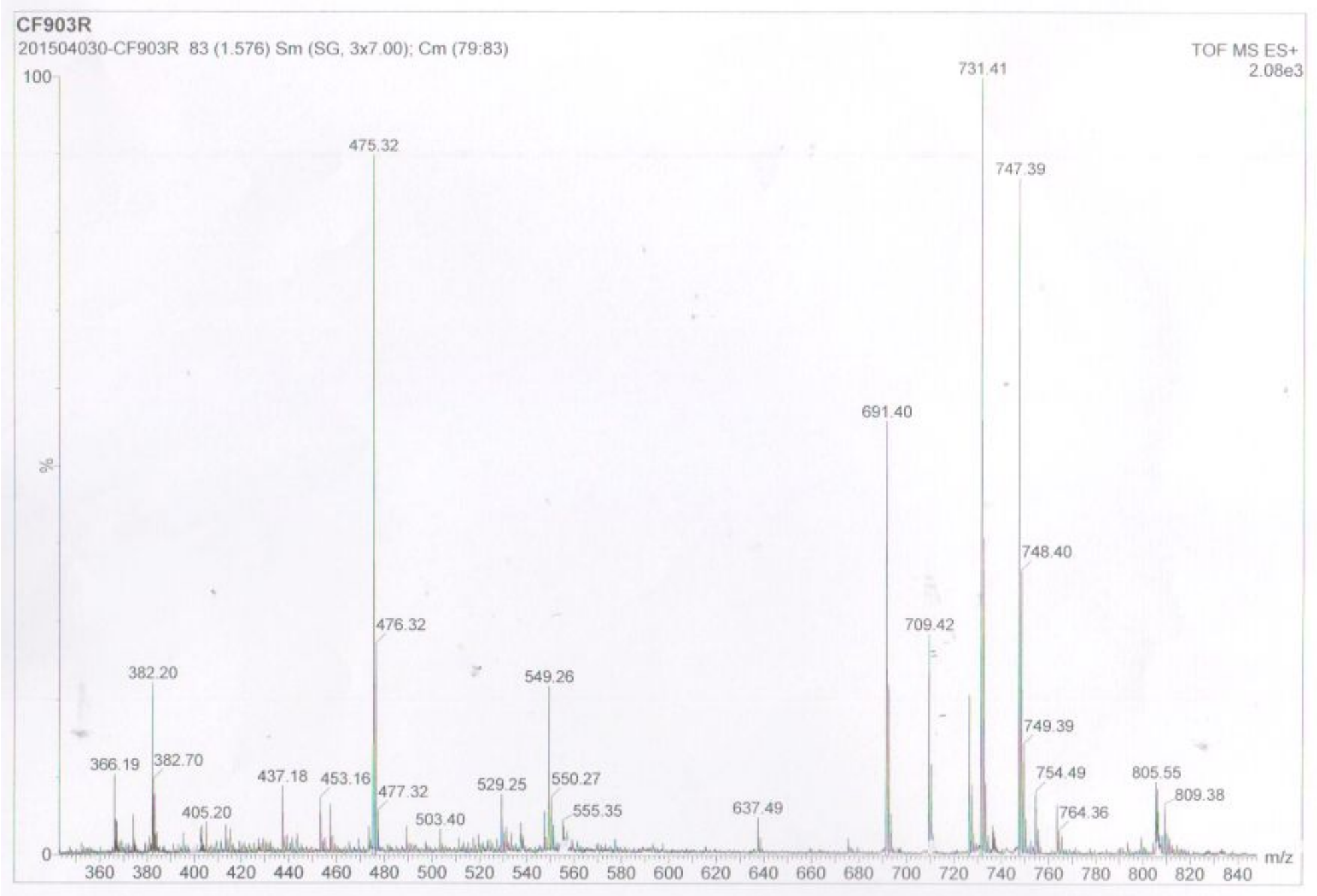

Figure S12. ESIMS spectrum of compound 1s 


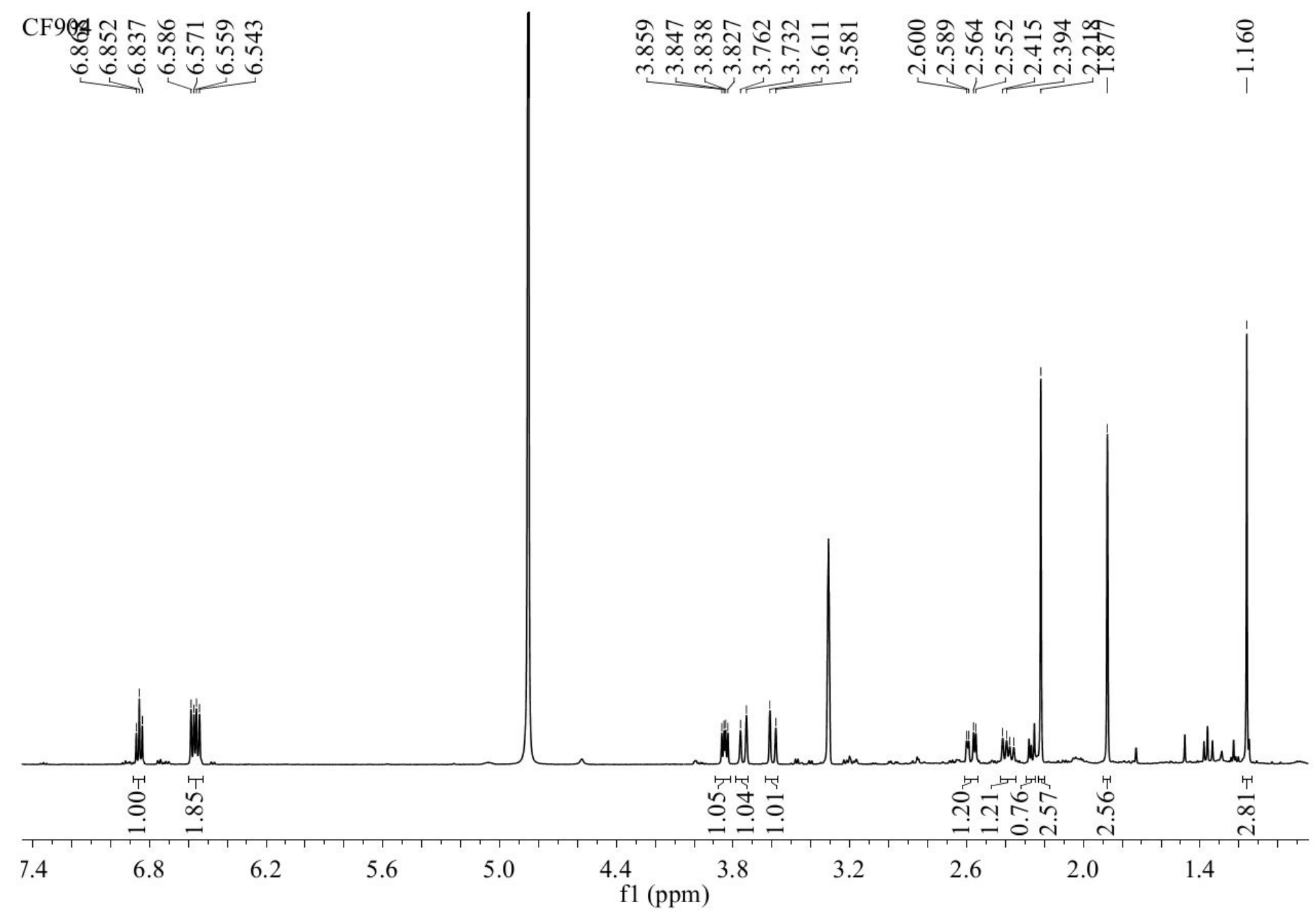

Figure S13. ${ }^{1} \mathrm{H}$ NMR (500 MHz, $\left.\mathrm{CD}_{3} \mathrm{OD}\right)$ spectrum of compound 2 


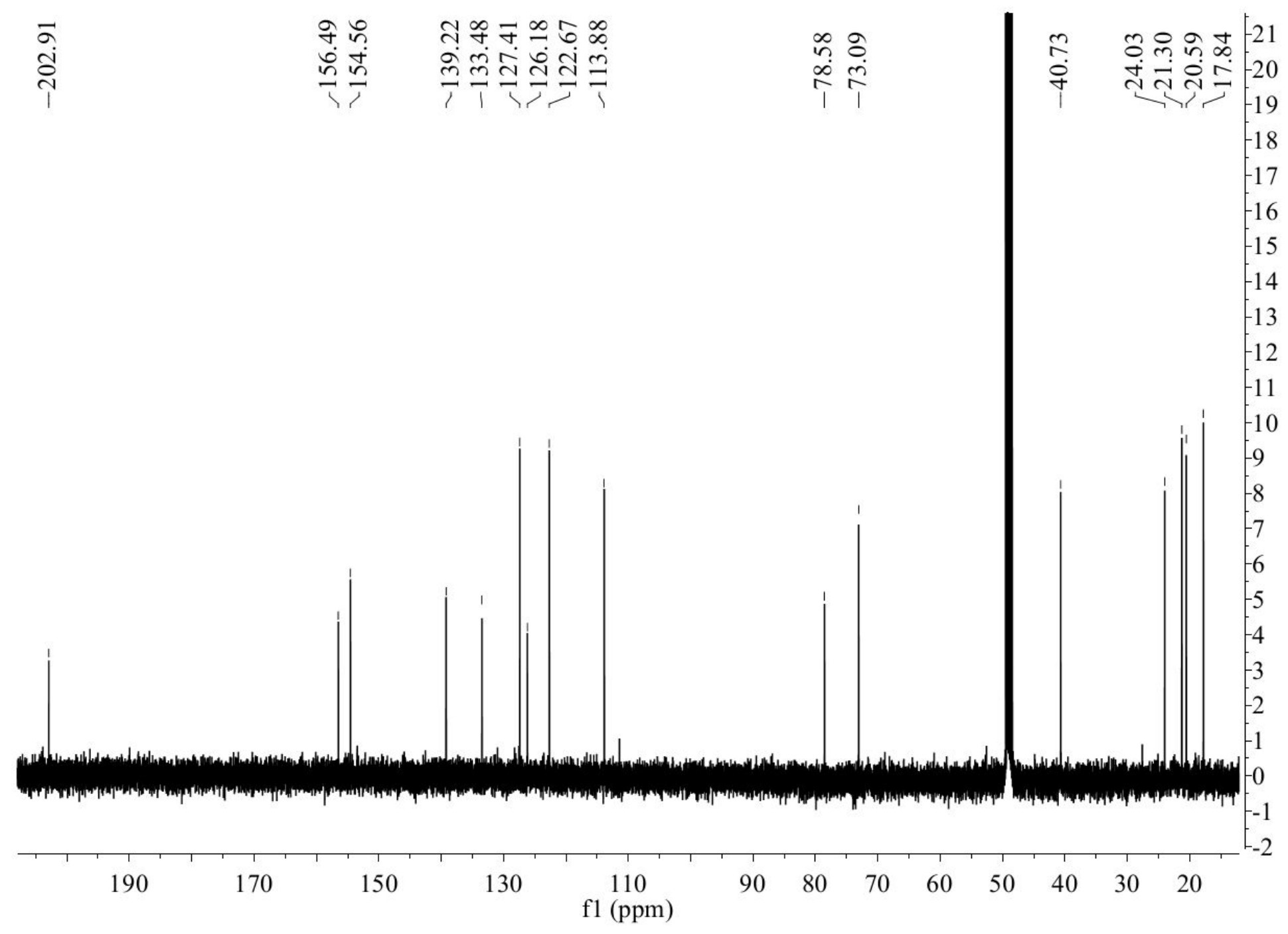

Figure S14. ${ }^{13} \mathrm{C}$ NMR (125 MHz, $\left.\mathrm{CD}_{3} \mathrm{OD}\right)$ spectrum of compound 2 


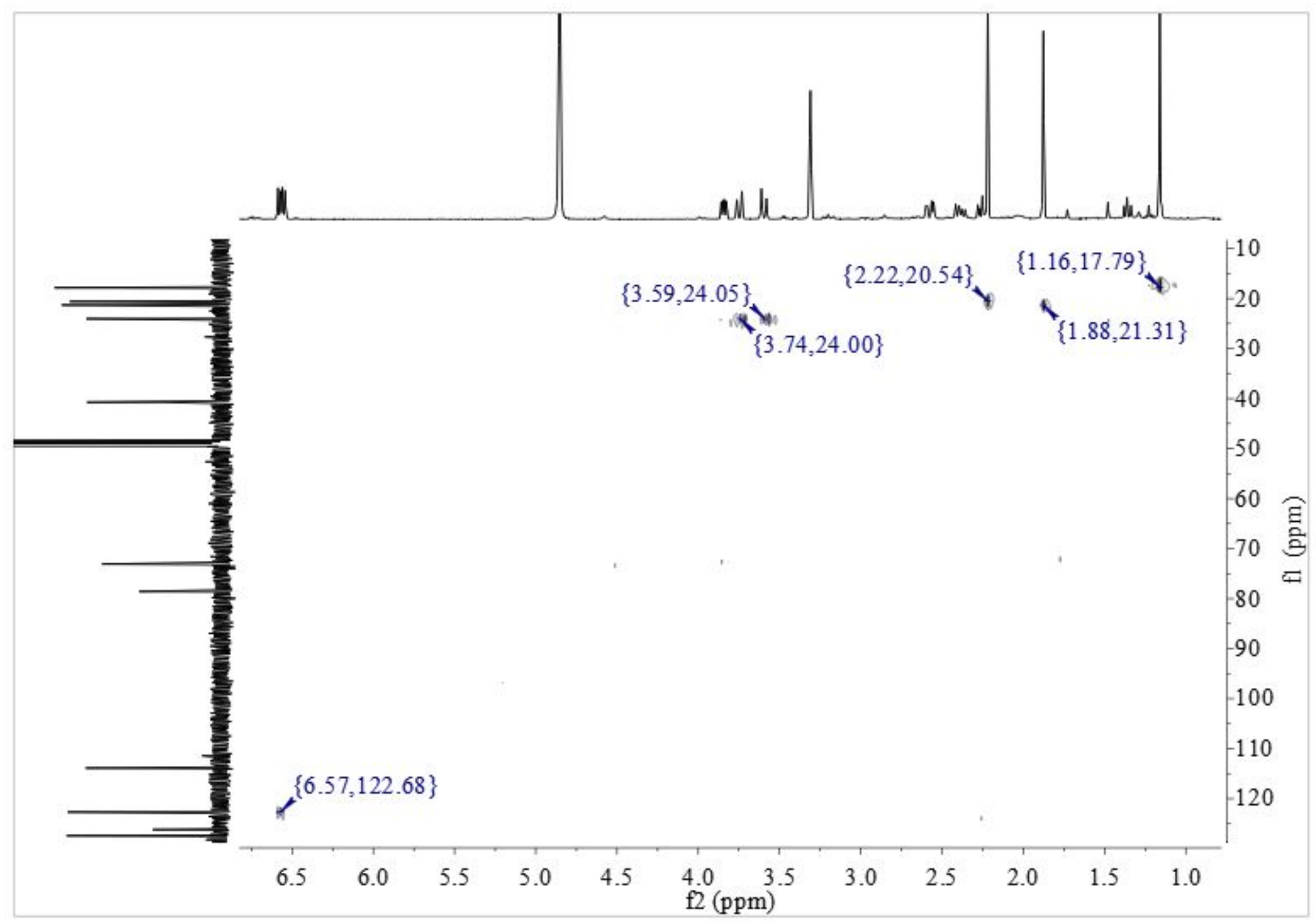

Figure S15. HSQC $\left(\mathrm{CD}_{3} \mathrm{OD}\right)$ spectrum of compound 2 


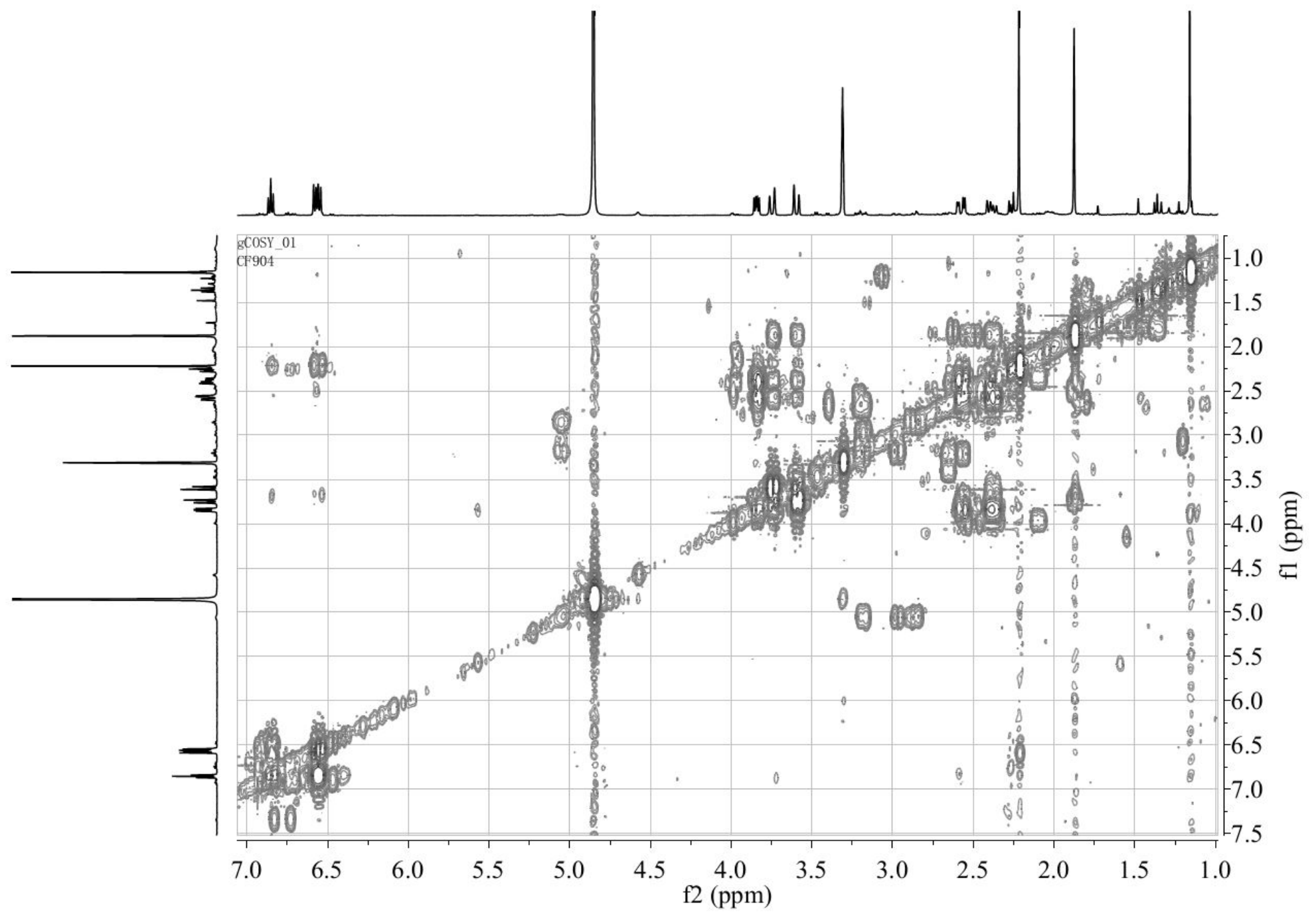

Figure S16. $\mathrm{COSY}\left(\mathrm{CD}_{3} \mathrm{OD}\right)$ spectrum of compound 2 


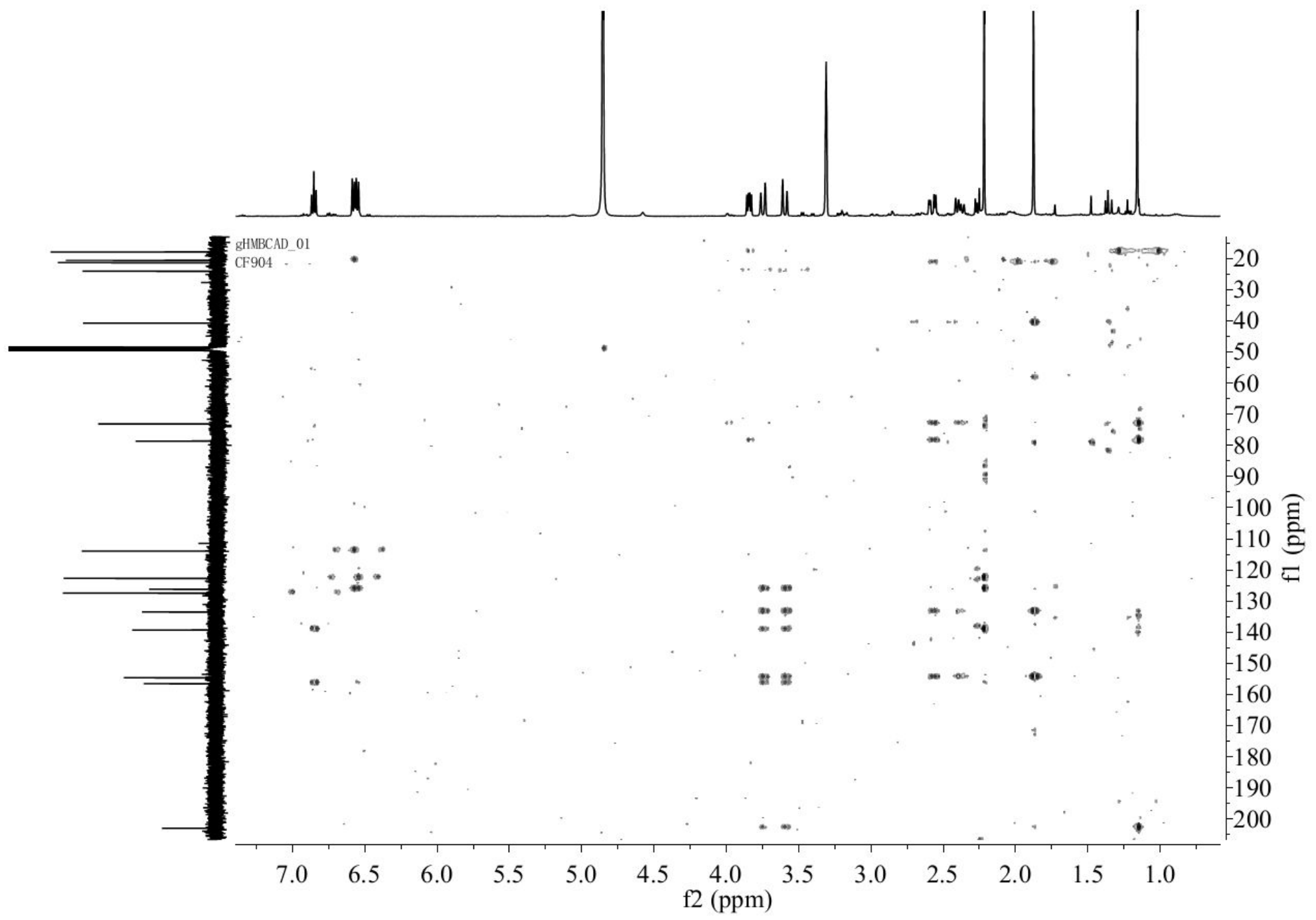

Figure S17. $\mathrm{HMBC}\left(\mathrm{CD}_{3} \mathrm{OD}\right)$ spectrum of compound 2 


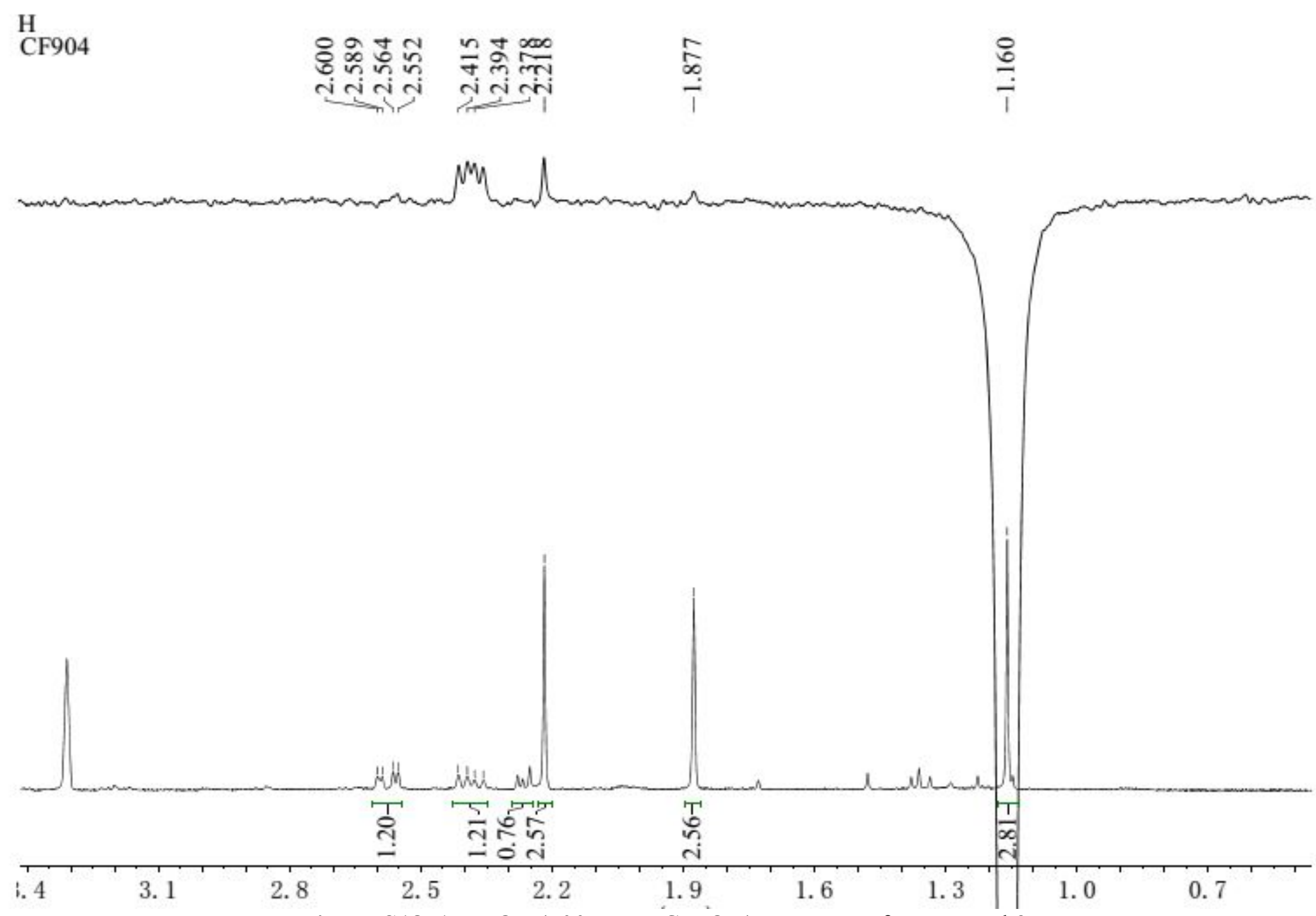

Figure S18. 1D NOE (500 MHz, $\left.\mathrm{CD}_{3} \mathrm{OD}\right)$ spectrum of compound 2 


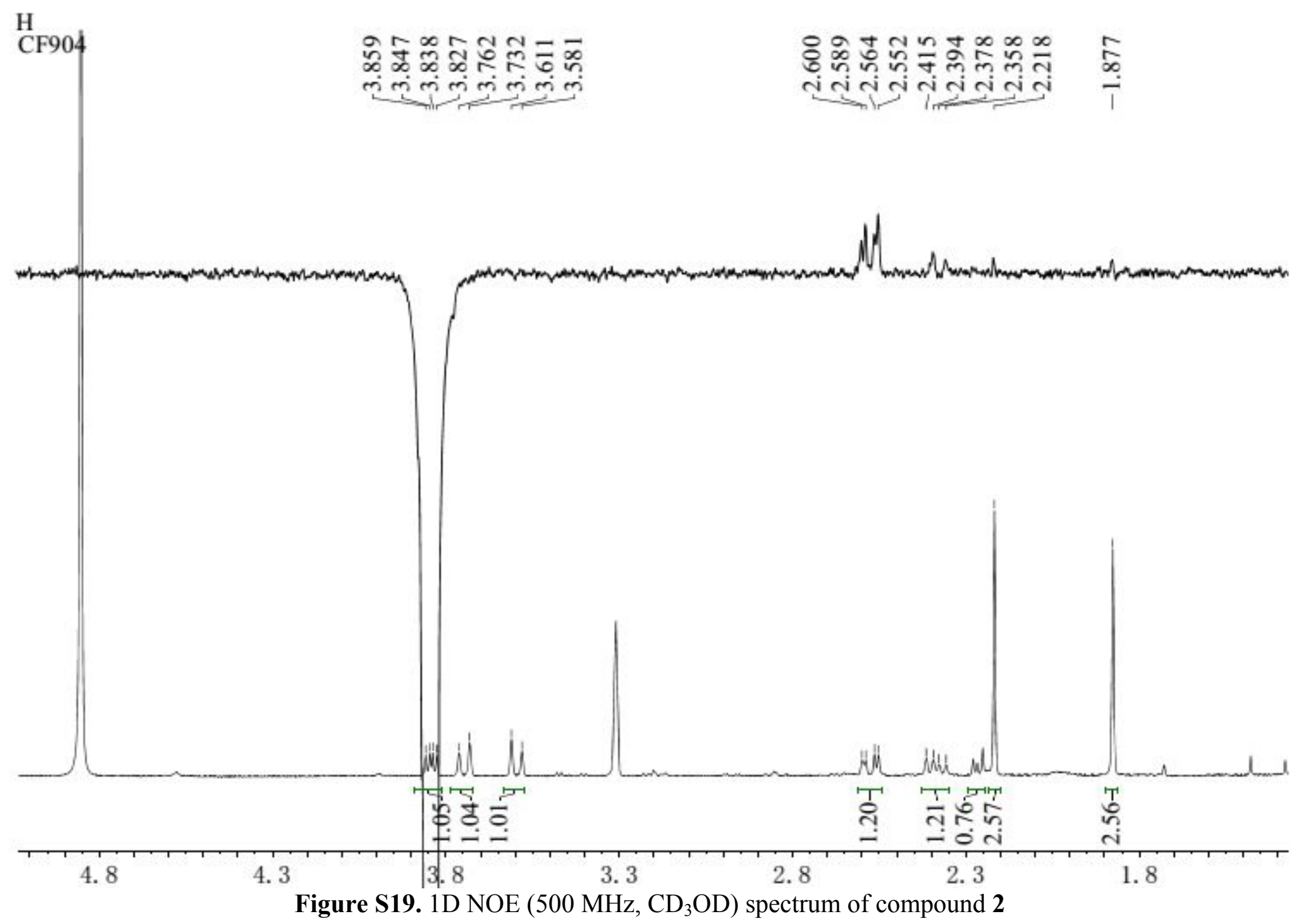


2015422-CF904 150422144655 \#137 RT: 1.09 AV: 1 SB: 16 0.01-0.13 NL: 4.59E6

T: FTMS + p ESI Full ms [100.00-2000.00]

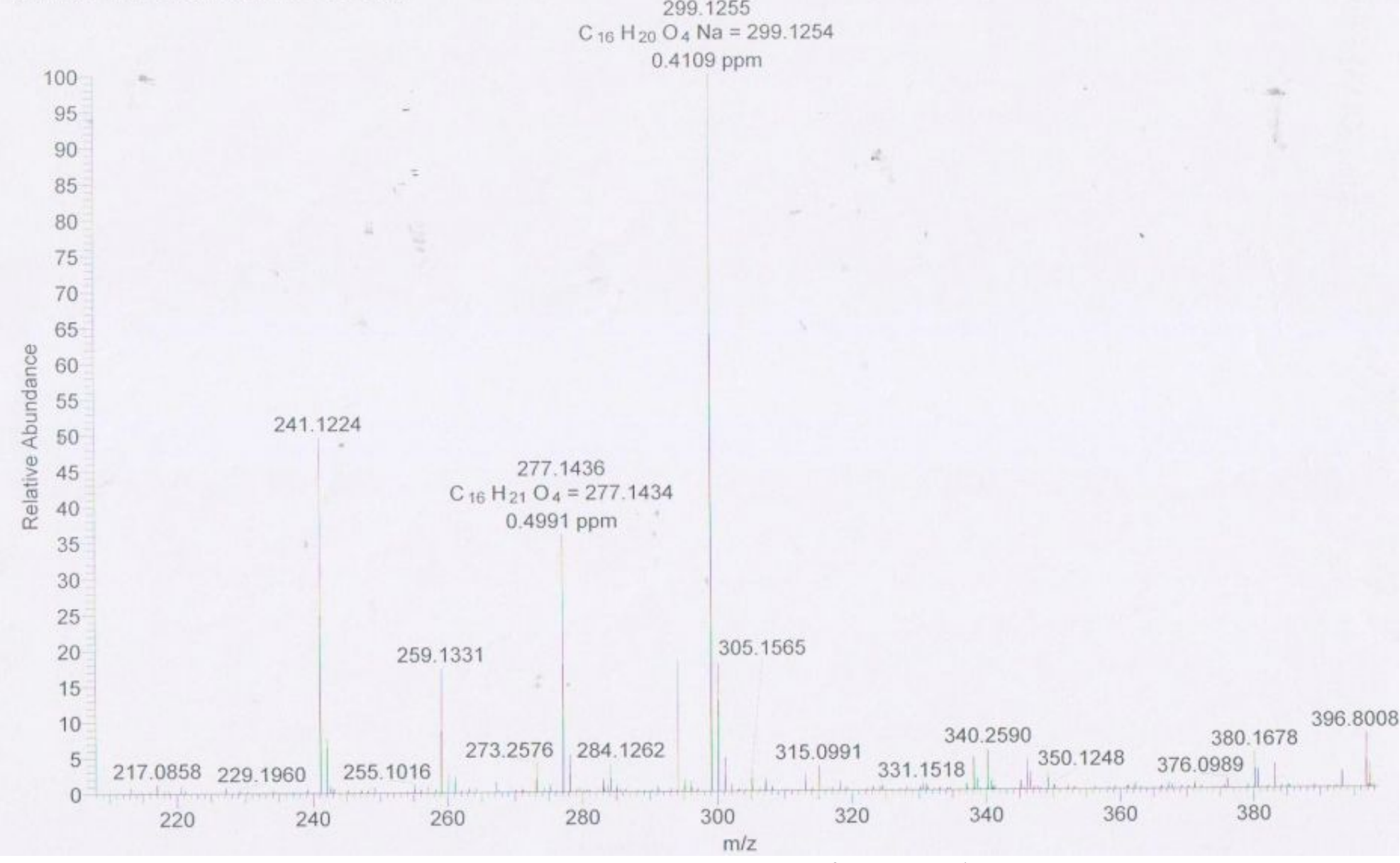

Figure S20. HRESIMS spectrum of compound 2 
Table S1. Experimental and calculated ${ }^{13} \mathrm{C}$ NMR data of $\mathbf{1}$ and $\mathbf{2}$

\begin{tabular}{cccccccccc}
\hline \multirow{2}{*}{ no } & \multicolumn{3}{c}{$\mathbf{1}$} & \multicolumn{3}{c}{$\mathbf{2}$} \\
\cline { 2 - 9 } & exptl. & calcd. & corr. & $|\Delta \delta|$ & exptl. & calcd. & corr. & $|\Delta \delta|$ \\
\hline 1 & 139.3 & 140.7 & 136.5 & 2.8 & 139.2 & 140.8 & 136.6 & 2.6 \\
2 & 122.7 & 123.4 & 120.0 & 2.7 & 122.7 & 123.5 & 120.1 & 2.6 \\
3 & 127.4 & 130.1 & 126.4 & 1.0 & 127.4 & 130.3 & 126.6 & 0.8 \\
4 & 113.9 & 120.2 & 117.0 & 3.1 & 113.9 & 120.2 & 116.9 & 3.0 \\
5 & 156.5 & 162.1 & 156.9 & 0.4 & 156.5 & 162.0 & 156.8 & 0.3 \\
6 & 126.3 & 127.6 & 124.1 & 2.2 & 126.2 & 127.1 & 123.6 & 2.6 \\
7 & 23.8 & 24.8 & 26.1 & 2.3 & 24.0 & 25.1 & 26.2 & 2.2 \\
8 & 132.8 & 136.1 & 132.2 & 0.6 & 133.5 & 137.3 & 133.3 & 0.2 \\
9 & 202.5 & 209.9 & 202.4 & 0.1 & 202.9 & 210.9 & 203.5 & 0.6 \\
10 & 77.3 & 77.9 & 76.7 & 0.6 & 78.6 & 79.6 & 78.2 & 0.4 \\
11 & 75.1 & 75.8 & 74.7 & 0.4 & 73.1 & 76.3 & 75.0 & 1.9 \\
12 & 39.8 & 37.6 & 38.3 & 1.5 & 40.7 & 38.5 & 39.0 & 1.7 \\
13 & 152.2 & 162.8 & 157.5 & 5.3 & 154.6 & 162.9 & 157.6 & 3.0 \\
14 & 20.6 & 19.7 & 21.3 & 0.7 & 20.6 & 19.7 & 21.1 & 0.5 \\
15 & 23.1 & 21.1 & 22.6 & 0.5 & 17.8 & 15.4 & 16.9 & 0.9 \\
16 & 21.2 & 20.2 & 21.8 & 0.6 & 21.3 & 20.1 & 21.4 & 0.1 \\
\hline
\end{tabular}


Table S2 The data of DP4+ method of compounds 1 and 2

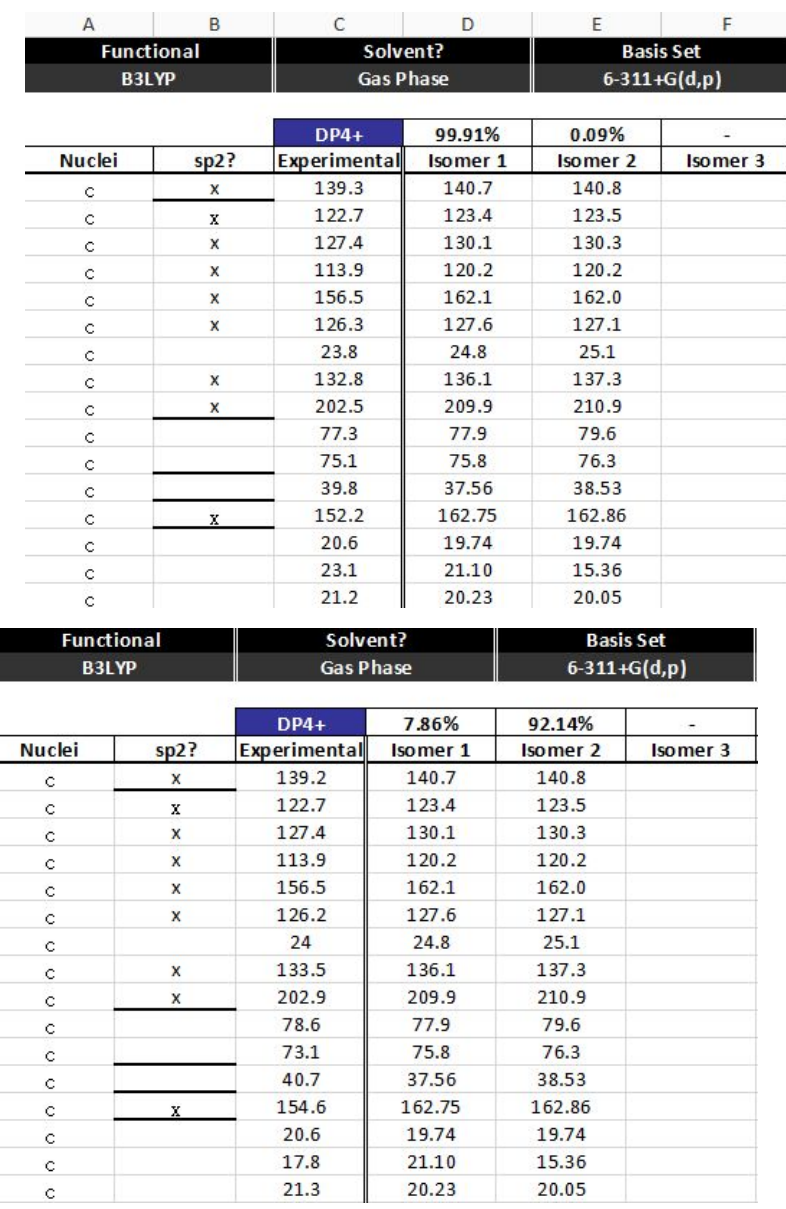


Table S3. The coordinate for the lowest-energy conformer [(10S,11S)-1] in ECD and ${ }^{13} \mathrm{C}$ NMR calculations

\begin{tabular}{|c|c|c|c|}
\hline \multicolumn{4}{|c|}{ Coordinates (Angstroms) } \\
\hline & $\mathrm{X}$ & $\mathrm{Y}$ & Z \\
\hline $\mathrm{C}$ & -3.74748700 & 0.70768900 & 1.10367800 \\
\hline $\mathrm{C}$ & -4.33135700 & -0.53739500 & 0.88004100 \\
\hline $\mathrm{C}$ & -3.73796100 & -1.43142200 & 0.00289100 \\
\hline $\mathrm{C}$ & -2.55658700 & -1.08190500 & -0.65655500 \\
\hline $\mathrm{C}$ & -1.96775500 & 0.18509600 & -0.46685800 \\
\hline $\mathrm{C}$ & -2.57905000 & 1.08198000 & 0.43610800 \\
\hline $\mathrm{C}$ & -0.75836800 & 0.60284500 & -1.30123500 \\
\hline $\mathrm{C}$ & 0.59340400 & 0.54626000 & -0.59943200 \\
\hline $\mathrm{C}$ & 1.06182500 & -0.79523100 & -0.21919100 \\
\hline $\mathrm{C}$ & 2.22542800 & -0.95171000 & 0.76595300 \\
\hline $\mathrm{C}$ & 3.32747500 & 0.05411800 & 0.40441800 \\
\hline $\mathrm{C}$ & 2.76804700 & 1.46430900 & 0.29047600 \\
\hline $\mathrm{C}$ & 1.43796800 & 1.59428200 & -0.42133700 \\
\hline $\mathrm{O}$ & -2.03911800 & -2.00934800 & -1.51473300 \\
\hline $\mathrm{O}$ & 0.55430700 & -1.82503500 & -0.66016300 \\
\hline $\mathrm{O}$ & 2.78780200 & -2.25008500 & 0.62259000 \\
\hline $\mathrm{C}$ & 1.70768600 & -0.76886900 & 2.20208400 \\
\hline $\mathrm{O}$ & 3.90362500 & -0.28078700 & -0.84942100 \\
\hline $\mathrm{C}$ & 1.18505300 & 2.99358000 & -0.90742800 \\
\hline $\mathrm{C}$ & -2.02137500 & 2.46658200 & 0.67116000 \\
\hline $\mathrm{H}$ & -4.21108400 & 1.40455200 & 1.79523500 \\
\hline $\mathrm{H}$ & -5.24521600 & -0.81115000 & 1.39806100 \\
\hline $\mathrm{H}$ & -4.15794500 & -2.41335800 & -0.18386700 \\
\hline $\mathrm{H}$ & -0.92892100 & 1.61026800 & -1.67832400 \\
\hline $\mathrm{H}$ & -0.71109800 & -0.03056400 & -2.19122600 \\
\hline $\mathrm{H}$ & 4.09795300 & 0.01928000 & 1.18756500 \\
\hline $\mathrm{H}$ & 2.66117300 & 1.90268900 & 1.29182100 \\
\hline $\mathrm{H}$ & 3.51307100 & 2.08261000 & -0.21980900 \\
\hline $\mathrm{H}$ & -1.06582400 & -1.99659000 & -1.45796800 \\
\hline $\mathrm{H}$ & 2.11036000 & -2.80979000 & 0.20790500 \\
\hline $\mathrm{H}$ & 1.26842800 & 0.21703200 & $2.3683650 \mathrm{C}$ \\
\hline $\mathrm{H}$ & 2.53484700 & -0.91058400 & 2.90110200 \\
\hline $\mathrm{H}$ & 0.94673400 & -1.52060100 & 2.42141900 \\
\hline $\mathrm{H}$ & 4.07529400 & -1.23380200 & -0.83292100 \\
\hline $\mathrm{H}$ & 1.38265800 & 3.71696300 & -0.10872200 \\
\hline $\mathrm{H}$ & 1.89148800 & 3.22821100 & -1.71230000 \\
\hline $\mathrm{H}$ & 0.18070100 & 3.16928800 & -1.28178400 \\
\hline $\mathrm{H}$ & -2.59628500 & 2.98924400 & 1.43772600 \\
\hline $\mathrm{H}$ & -0.97830700 & 2.44577900 & 0.99770100 \\
\hline $\mathrm{H}$ & -2.06264300 & 3.08128900 & -0.23480000 \\
\hline
\end{tabular}


Table S4. The coordinate for the lowest-energy conformer $[(10 S, 11 R)-1]$ in ECD and ${ }^{13} \mathrm{C}$ NMR calculations

\begin{tabular}{|c|c|c|c|}
\hline \multicolumn{4}{|c|}{ Coordinates (Angstroms) } \\
\hline & $\mathrm{X}$ & $\mathrm{Y}$ & $\mathrm{Z}$ \\
\hline $\mathrm{C}$ & -3.67343300 & 0.71714900 & 1.31006000 \\
\hline $\mathrm{C}$ & -4.29637100 & -0.51202200 & 1.10477600 \\
\hline $\mathrm{C}$ & -3.79065400 & -1.39482100 & 0.16365900 \\
\hline $\mathrm{C}$ & -2.65856600 & -1.04945600 & -0.57886500 \\
\hline $\mathrm{C}$ & -2.03401100 & 0.20286100 & -0.40831600 \\
\hline $\mathrm{C}$ & -2.55475500 & 1.08805500 & 0.56070700 \\
\hline $\mathrm{C}$ & -0.89105700 & 0.62232800 & -1.33088900 \\
\hline $\mathrm{C}$ & 0.51376900 & 0.53228100 & -0.74742800 \\
\hline $\mathrm{C}$ & 0.98565500 & -0.82762500 & -0.42401500 \\
\hline $\mathrm{C}$ & 2.22077100 & -1.00461000 & 0.45872500 \\
\hline $\mathrm{C}$ & 3.28971900 & -0.02942700 & -0.05453600 \\
\hline $\mathrm{C}$ & 2.77302500 & 1.39821900 & -0.03475700 \\
\hline $\mathrm{C}$ & 1.38662200 & 1.56632900 & -0.61745300 \\
\hline $\mathrm{O}$ & -2.22658500 & -1.96450100 & -1.49611300 \\
\hline $\mathrm{O}$ & 0.43560100 & -1.84126100 & -0.85015500 \\
\hline $\mathrm{O}$ & 2.74789400 & -2.31519900 & 0.30659900 \\
\hline $\mathrm{C}$ & 1.83586200 & -0.78451300 & 1.93193100 \\
\hline $\mathrm{O}$ & 4.48087700 & -0.08696100 & 0.71116600 \\
\hline $\mathrm{C}$ & 1.10905500 & 2.98114400 & -1.04299500 \\
\hline $\mathrm{C}$ & -1.95380100 & 2.45643700 & 0.78270000 \\
\hline $\mathrm{H}$ & -4.06773900 & 1.40448300 & 2.05222700 \\
\hline $\mathrm{H}$ & -5.17146800 & -0.78271200 & 1.68727400 \\
\hline $\mathrm{H}$ & -4.24209500 & -2.36495400 & -0.01083100 \\
\hline $\mathrm{H}$ & -0.92795700 & 0.00610100 & -2.23351200 \\
\hline $\mathrm{H}$ & -1.07827300 & 1.63910700 & -1.67373600 \\
\hline $\mathrm{H}$ & 3.50578200 & -0.32384800 & -1.09183400 \\
\hline $\mathrm{H}$ & 3.47750800 & 2.04629900 & -0.56556300 \\
\hline $\mathrm{H}$ & 2.77066100 & 1.76182900 & 1.0015440 \\
\hline $\mathrm{H}$ & -1.25190900 & -1.97216400 & -1.51301800 \\
\hline $\mathrm{H}$ & 2.05542800 & -2.86516800 & -0.09437500 \\
\hline $\mathrm{H}$ & 1.39178100 & 0.19694700 & 2.1104770 \\
\hline $\mathrm{H}$ & 2.72784100 & -0.88256000 & 2.55137300 \\
\hline $\mathrm{H}$ & 1.11489000 & -1.54557900 & 2.23665700 \\
\hline $\mathrm{H}$ & 4.81668500 & -0.99246800 & 0.66748100 \\
\hline $\mathrm{H}$ & 1.37553000 & 3.67685400 & -0.23993300 \\
\hline $\mathrm{H}$ & 0.07949600 & 3.17365300 & -1.32977000 \\
\hline $\mathrm{H}$ & 1.75015600 & 3.24018900 & -1.89372700 \\
\hline $\mathrm{H}$ & -2.05403600 & 3.09382400 & -0.10278100 \\
\hline $\mathrm{H}$ & -2.45638100 & 2.97035200 & 1.60397900 \\
\hline $\mathrm{H}$ & -0.88918500 & 2.40922100 & 1.02627600 \\
\hline
\end{tabular}


Table S5. The coordinate for the lowest-energy conformer $[(10 R, 11 S)-1]$ in ECD calculation

\begin{tabular}{|c|c|c|c|}
\hline & \multicolumn{2}{|c|}{ Coordinates (Angstroms) } & \multirow[b]{2}{*}{$\mathrm{Z}$} \\
\hline & $\mathrm{X}$ & $\mathrm{Y}$ & \\
\hline $\mathrm{C}$ & 3.67324200 & 0.71721600 & 1.31028900 \\
\hline $\mathrm{C}$ & 4.29643200 & -0.51181000 & 1.10491700 \\
\hline $\mathrm{C}$ & 3.79100900 & -1.39454000 & 0.16351500 \\
\hline $\mathrm{C}$ & 2.65902100 & -1.04918500 & -0.57911700 \\
\hline $\mathrm{C}$ & 2.03411200 & 0.20292700 & -0.40836000 \\
\hline $\mathrm{C}$ & 2.55454800 & 1.08804100 & 0.56088200 \\
\hline $\mathrm{C}$ & 0.89106100 & 0.62216500 & -1.33084000 \\
\hline $\mathrm{C}$ & -0.51375200 & 0.53206300 & -0.74733000 \\
\hline $\mathrm{C}$ & -0.98556600 & -0.82771300 & -0.42356400 \\
\hline $\mathrm{C}$ & -2.22096800 & -1.00457100 & 0.45882500 \\
\hline $\mathrm{C}$ & -3.28979400 & -0.02954900 & -0.05492000 \\
\hline $\mathrm{C}$ & -2.77313100 & 1.39812500 & -0.03504000 \\
\hline $\mathrm{C}$ & -1.38663700 & 1.56618600 & -0.61757900 \\
\hline $\mathrm{O}$ & 2.22742200 & -1.96397600 & -1.49693600 \\
\hline $\mathrm{O}$ & -0.43528400 & -1.84155200 & -0.84899600 \\
\hline $\mathrm{O}$ & -2.74790200 & -2.31521000 & 0.30679100 \\
\hline $\mathrm{C}$ & -1.83634700 & -0.78414000 & 1.93209700 \\
\hline $\mathrm{O}$ & -4.48132500 & -0.08718400 & 0.71013100 \\
\hline $\mathrm{C}$ & -1.10902400 & 2.98100600 & -1.04312000 \\
\hline $\mathrm{C}$ & 1.95327500 & 2.45626100 & 0.78305200 \\
\hline $\mathrm{H}$ & 4.06731500 & 1.40452100 & 2.05261500 \\
\hline $\mathrm{H}$ & 5.17148900 & -0.78243200 & 1.68751100 \\
\hline $\mathrm{H}$ & 4.24265200 & -2.36455200 & -0.01113300 \\
\hline $\mathrm{H}$ & 1.07808900 & 1.63893400 & -1.67384800 \\
\hline $\mathrm{H}$ & 0.92797900 & 0.00578800 & -2.23336700 \\
\hline $\mathrm{H}$ & -3.50524100 & -0.32398700 & -1.09235200 \\
\hline $\mathrm{H}$ & -2.77084500 & 1.76170100 & 1.00127100 \\
\hline $\mathrm{H}$ & -3.47754000 & 2.04628400 & -0.56585900 \\
\hline $\mathrm{H}$ & 1.25280900 & -1.97245900 & -1.51329500 \\
\hline $\mathrm{H}$ & -2.05500200 & -2.86541400 & -0.09307800 \\
\hline $\mathrm{H}$ & -1.39216000 & 0.19728700 & 2.11060900 \\
\hline $\mathrm{H}$ & -2.72846600 & -0.88200900 & 2.55135800 \\
\hline $\mathrm{H}$ & -1.11552500 & -1.54527100 & 2.23706200 \\
\hline $\mathrm{H}$ & -4.81636600 & -0.99301100 & 0.66729000 \\
\hline $\mathrm{H}$ & -1.37524000 & 3.67664100 & -0.23988800 \\
\hline $\mathrm{H}$ & -0.07951900 & 3.17349500 & -1.33011300 \\
\hline $\mathrm{H}$ & -1.75034200 & 3.24022000 & -1.89363400 \\
\hline $\mathrm{H}$ & 0.88872100 & 2.40874000 & 1.02688000 \\
\hline $\mathrm{H}$ & 2.45588900 & 2.97029900 & 1.60424200 \\
\hline $\mathrm{H}$ & 2.05313400 & 3.09370900 & -0.10243300 \\
\hline
\end{tabular}


Table S6. The coordinate for the lowest-energy conformer $[(10 R, 11 R)-1]$ in ECD calculation

\begin{tabular}{|c|c|c|c|}
\hline & \multicolumn{2}{|c|}{ Coordinates (Angstroms) } & \multirow[b]{2}{*}{$\mathrm{Z}$} \\
\hline & $\mathrm{X}$ & $\mathrm{Y}$ & \\
\hline $\mathrm{C}$ & 3.74713900 & 0.70787400 & 1.10412000 \\
\hline $\mathrm{C}$ & 4.33138500 & -0.53700500 & 0.88037600 \\
\hline $\mathrm{C}$ & 3.73842100 & -1.43098800 & 0.00285900 \\
\hline $\mathrm{C}$ & 2.55716200 & -1.08158500 & -0.65679300 \\
\hline $\mathrm{C}$ & 1.96782600 & 0.18514800 & -0.46690200 \\
\hline $\mathrm{C}$ & 2.57871600 & 1.08200200 & 0.43639600 \\
\hline $\mathrm{C}$ & 0.75840800 & 0.60271100 & -1.30131700 \\
\hline $\mathrm{C}$ & -0.59344500 & 0.54610100 & -0.59961800 \\
\hline $\mathrm{C}$ & -1.06182900 & -0.79532800 & -0.21915000 \\
\hline $\mathrm{C}$ & -2.22546700 & -0.95160900 & 0.76613800 \\
\hline $\mathrm{C}$ & -3.32763900 & 0.05391600 & 0.40427200 \\
\hline $\mathrm{C}$ & -2.76834200 & 1.46414100 & 0.28965300 \\
\hline $\mathrm{C}$ & -1.43814100 & 1.59410700 & -0.42190300 \\
\hline $\mathrm{O}$ & 2.04028000 & -2.00897800 & -1.51545600 \\
\hline $\mathrm{O}$ & -0.55414400 & -1.82522500 & -0.65962300 \\
\hline $\mathrm{O}$ & -2.78755000 & -2.25015600 & 0.62313600 \\
\hline $\mathrm{C}$ & -1.70777900 & -0.76819900 & 2.20217400 \\
\hline $\mathrm{O}$ & -3.90403000 & -0.28170400 & -0.84923600 \\
\hline $\mathrm{C}$ & -1.18526400 & 2.99340300 & -0.90798600 \\
\hline $\mathrm{C}$ & 2.02067400 & 2.46639900 & 0.67170000 \\
\hline $\mathrm{H}$ & 4.21040700 & 1.40470800 & 1.79593100 \\
\hline $\mathrm{H}$ & 5.24518200 & -0.81064600 & 1.39856700 \\
\hline $\mathrm{H}$ & 4.15870600 & -2.41277600 & -0.18401500 \\
\hline $\mathrm{H}$ & 0.71117800 & -0.03077000 & -2.19126200 \\
\hline $\mathrm{H}$ & 0.92889300 & 1.61010800 & -1.67851600 \\
\hline $\mathrm{H}$ & -4.09796400 & 0.01946500 & 1.18758000 \\
\hline $\mathrm{H}$ & -3.51336100 & 2.08207600 & -0.22109300 \\
\hline $\mathrm{H}$ & -2.66185300 & 1.90310700 & 1.29080100 \\
\hline $\mathrm{H}$ & 1.06709900 & -1.99675800 & -1.45873600 \\
\hline $\mathrm{H}$ & -2.10991400 & -2.80982300 & 0.20878900 \\
\hline $\mathrm{H}$ & -1.26875400 & 0.21786300 & 2.36818000 \\
\hline $\mathrm{H}$ & -2.53487400 & -0.90985000 & 2.90128500 \\
\hline $\mathrm{H}$ & -0.94664200 & -1.51966700 & 2.42180300 \\
\hline $\mathrm{H}$ & -4.07492700 & -1.23483000 & -0.83247200 \\
\hline $\mathrm{H}$ & -0.18157100 & 3.16858200 & -1.28434800 \\
\hline $\mathrm{H}$ & -1.38060900 & 3.71652800 & -0.10846000 \\
\hline $\mathrm{H}$ & -1.89321400 & 3.22905300 & -1.71120300 \\
\hline $\mathrm{H}$ & 2.06177300 & 3.08128800 & -0.23413800 \\
\hline $\mathrm{H}$ & 0.97762000 & 2.44523200 & 0.99825400 \\
\hline $\mathrm{H}$ & 2.59543300 & 2.98909100 & 1.43836600 \\
\hline
\end{tabular}

